\title{
A Rare Soft-Tissue Tumor in a 15-Year-Old Boy With Tuberous Sclerosis Complex: Answer
}

\author{
Lindsey Oudijk, $M D, P h D,{ }^{*}$ Elodie J. Mendels, MD, $\dagger$ and Jeffrey Damman, $M D, P h D^{*}$
}

(Am J Dermatopathol 2020;42:460)

\section{(Continued from page e66)}

\section{ANSWER}

This is a folliculocystic and collagen hamartoma (FCCH), a rare benign collagen hamartoma strongly associated with tuberous sclerosis complex (TSC). Hamartomas in TSC with mutations in the TSCl (encodes for hamartin) and TSC2 (tuberin) genes have high mTORC1 activity as a result of lack of inhibition by this TSC tumor suppressor complex. High mTORC1 activity stimulates proliferation of fibroblasts, keratinocytes, hair follicles, and sebaceous glands. ${ }^{1}$ However, for the development of hamartomas, a second somatic event is required, in addition to the germline TSC1/TSC2 mutation. ${ }^{2}$

\section{DISCUSSION}

TSC is an autosomal dominant disorder caused by mutations in the TSC1 $(30 \%)$ or TSC2 $(65 \%)$ gene. ${ }^{2}$ In $5 \%-$ $10 \%$ of TSC patients, no mutation can be identified, and some patients have low-level mosaicism. ${ }^{2}$ For detailed information about the diagnostic and genetic criteria, we refer to the International Tuberous Sclerosis Complex Consensus conference, held in 2012.

FCCH was suggested as a new type of complex hamartoma related with TSC by Torrelo et al in $2012 .{ }^{3}$ They described 6 male patients with congenital/early childhood skin lesions consisting of large plaques with an irregular surface that became scattered with numerous comedo-like openings over time. Five of the 6 patients had a definite clinical diagnosis of TSC. After this initial description, only 4 additional case reports have been published, including 3 female patients ${ }^{1,4,5}$ and 1 patient without TSC. ${ }^{6}$

FCCH histopathologically resembles angiofibroma, shagreen patches, and TSC-unrelated collagen nevi. These lesions have in common that they show coarse, rather than delicate, collagen fibers, which are often haphazardly arranged through the dermis. In contrast to the previously mentioned lesion, FCCH shows collagen fibers that are arranged in a concentric array around hair follicles. This perifollicular fibrosis is often associated with some distortion of the pilosebaceous units. Furthermore, infundibular cyst formation is a characteristic feature, which can only be observed in FCCH. ${ }^{6}$ Clinically, the case might also resemble plexiform neurofibroma (in NF1) or nevus sebaceous. In nevus sebaceous, the appearance of sebaceous gland hyperplasia with or without immature hair follicles in the dermis is the key feature. This is in contrast to the mature hair follicles surrounded by fibrosis and inflammation as can be seen in FCCH. Finally, fibrous hamartoma of infancy is a rare softtissue lesion that may enter the differential diagnosis. This lesion harbors a characteristic triphasic morphology of fat, mesenchymal tissue, and fibroblastic fascicles. ${ }^{4}$

The final diagnosis of FCCH requires familiarity with the clinical features and the 3 histopathological features of this lesion. Likely, FCCH is underrecognized because not many clinicians and pathologist are familiar with this relatively new entity. When $\mathrm{FCCH}$ is the presenting feature of TSC, early recognition is important for further clinical workup leading to a final diagnosis. In patients already diagnosed with TSC, early recognition of this benign entity within the spectrum of TSC could prevent distress and anxiety during the diagnostic process. Altogether, we think it is important to recognize this rare, relatively new entity and to further study its relation to TSC.

\section{REFERENCES}

1. Brown MM, Walsh EJ, Yu L, et al. Progressive scalp plaque in a girl with tuberous sclerosis. Pediatr Dermatol. 2014;31:249-250.

2. Peron A, Au KS, Northrup H. Genetics, genomics, and genotypephenotype correlations of TSC: insights for clinical practice. Am J Med Genet C Semin Med Genet. 2018;178:281-290.

3. Torrelo A, Hadj-Rabia S, Colmenero I, et al. Folliculocystic and collagen hamartoma of tuberous sclerosis complex. J Am Acad Dermatol. 2012;66: 617-621.

4. Kaplan L, Kazlouskaya V, Ugorji R, et al. Folliculocystic and collagen hamartoma of tuberous sclerosis: a new case in a female patient and review of literature. J Cutan Pathol. 2018;45:67-70.

5. Bishnoi A, Tripathy S, Vinay K, et al. Image Gallery: folliculocystic and collagen hamartoma: a lesser-known presentation of tuberous sclerosis. $\mathrm{Br}$ J Dermatol. 2018;178:e276.

6. An JM, Kim YS, Park YL, et al. Folliculocystic and collagen hamartoma: a new entity? Ann Dermatol. 2015;27:593-596.

From the Departments of *Pathology, and $†$ Pediatric Dermatology, Erasmus MC, University Medical Center, Rotterdam, the Netherlands.

The authors declare no conflicts of interest.

Correspondence: L. Oudijk, Department of Pathology, Erasmus University Medical Center Rotterdam, Doctor Molewaterplein 40, 3015 GD

Rotterdam, the Netherlands (e-mail: 1.oudijk@erasmusmc.nl).

Copyright (C) 2020 Wolters Kluwer Health, Inc. All rights reserved. 